Wenn Aufstehen zum Albtraum wird

\title{
COPD-assoziierte Schlafstörungen reduzieren Lebensqualität und erhöhen Mortalität
}

- „Der Start in den Tag ist ein Albtraum für mich, Duschen und Anziehen fällt mir extrem schwer. Aber nach einer Stunde etwa geht es mir besser und ich komme ganz gut durch den Tag." Diese typische Aussage eines COPD-Patienten verdeutlicht, dass die Beschwerdesymptomatik bei COPD ungleich über den Tag verteilt ist: Husten, Auswurf, Atemnot und Übermüdung aufgrund einer schlechten Nacht machen vielen COPD-Patienten besonders in den frühen Morgenstunden zu schaffen, berichtete Prof. Peter Calverley, Liverpool.

Dass COPD-Patienten v. a. nachts und früh morgens mit Beschwerden kämpfen, veranschaulicht die Arbeit von Partridge et al. (CMRO 2009, s. Abb. 1). Inzwischen liegen zahlreiche Literaturen vor, die den $\mathrm{Zu}$ sammenhang zwischen COPD, Schlafstörungen und erhöhter Morbidität bestätigen:

\section{Schlafstörungen erhöhen Exazerbationsrisiko}

Eine Arbeitsgruppe aus San Francisco (Omachi TA, et al. Sleep Med. 2012; 13 (5): 476-83) untersuchte bei 91 COPD-Patienten, inwiefern krankheitsassoziierte Schlafstörungen den Krankheitsverlauf beeinflussen. Ergebnis: Schlafstörungen werden mit zunehmender COPD-Krankheitsprogredienz häufiger. Sie sind ein unabhängiger Risikofaktor für COPD-Exazerbationen, Notfallbehandlungen und für die Mortalität.

\section{Nächtliche Symptome mindern Lebensqualität}

In einer Studie von Scharf SM (Internat J COPD 2011; 6: 1-12) wurde bei 180 COPDPatienten die Häufigkeit von Schlafstörungen sowie deren Einfluss auf die Lebensqualität untersucht. Ergebnis: $77 \%$ der Patienten berichteten Schlafprobleme (medianer Pittsburgh Sleep Quality Index 12), die eindeutig die allgemeine und die krankheitsspezifische Lebensqualität minderten.

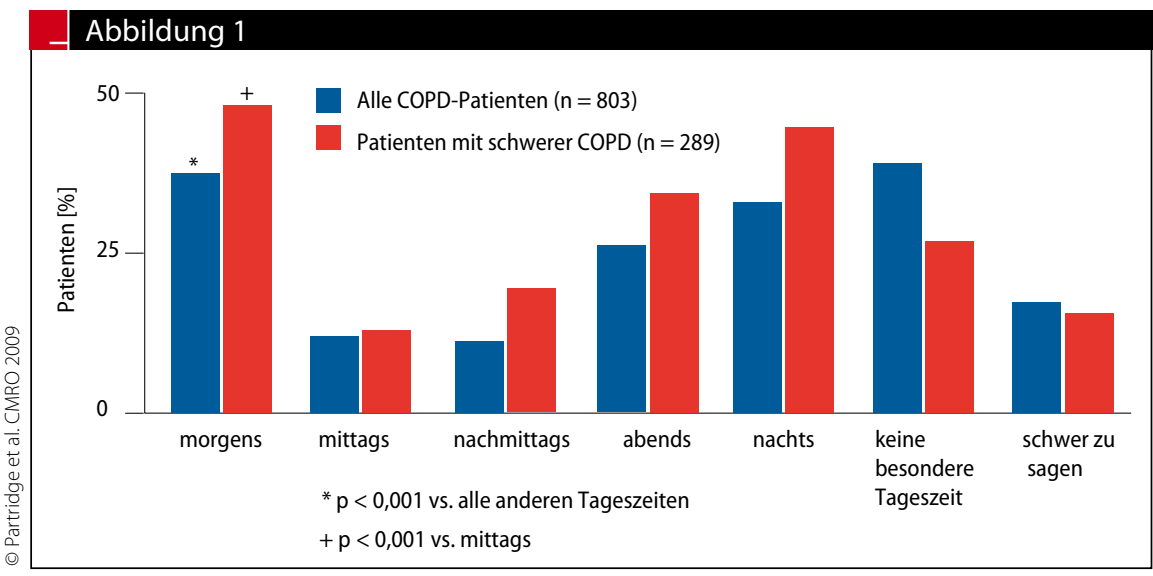

Abb. 1 Die Beschwerdesymptomatik ist bei vielen COPD-Patienten nachts und morgens am ausgeprägtesten.

\section{Beschwerdekumulation in den Morgenstunden}

In einer Studie aus der Türkei (Tüberküloz ve Toraks Dergisi 2011; 59 (4): 328-339) charakterisierten die Autoren die Beschwerdevariabilität von 514 COPD-Patienten. Nach ihren Befunden leiden 41$61 \%$ der Patienten, insbesondere in den frühen Morgenstunden, an Husten, Auswurf und Atemnot. Treppensteigen, Schuhe und Strümpfe anziehen sowie Duschen und Waschen waren dadurch deutlich eingeschränkt.

Eine bessere nächtliche und frühmorgendliche Symptomkontrolle könnte der neue langwirksame Muskarin-RezeptorAntagonist (LAMA) Aclidinium (Bretaris ${ }^{\circledR}$ Genuair $^{\circledast}$ ) versprechen. Laut Prof. Heinrich Worth, Fürth, zeichnet sich der Wirkstoff durch eine hohe Affinität und Verweildauer am relevanten M3-Rezeptor aus. Die zweimal tägliche Inhalation $(2 \times 400 \mu \mathrm{g} / \mathrm{d})$ führte in einer Vergleichsstudie gegenüber dem einmal täglich verabreichten Tiotropium zu einer besseren Symptomkontrolle (Husten, Dypspnoe) im Intervall 12-24 Stunden nach der ersten Dosierung. Aclidinium ist deshalb eine gute Therapieoption für Patienten mit nächtlichen und morgendlichen Beschwerden, so Worth.

- Dr. med. Dirk Einecke
- Quellen: a.) Satelliten-Symposium „Seeing COPD from the patient's perspective" (Veranstalter: Almirall, Menarini) im Rahmen der Jahrestagung der European Respiratory Society, Wien, September 2012; b.) Pressekonferenz „Bretaris ${ }^{\circledast}$ Genuair $^{\circledR}$ - der neue langwirksame Antagonist muskarinischer Acetylcholinrezeptoren (LAMA) in der COPD-Therapie", Berlin, Oktober 2012 (Veranstalter: Berlin-Chemie)

\section{Kurz notiert}

Hautschonendes Gel zur Händedesinfektion - Gemäß den Empfehlungen des Robert Koch-Instituts basiert das neue ROGG-Desinfektionsgel auf alkoholischen Wirkstoffen. Einzigartig ist jedoch die Galenik: Zum Schutz der Haut sind die bakteriziden, fungiziden und begrenzt viruziden (bei Noro-Viren wirksam) Alkohole in ein Gel mit rückfettenden Eigenschaften gebunden. Dadurch werden Hautschäden wie Risse und Schrunden an den Händen vermieden und das Desinfektionsgel ist damit auch für den Dauereinsatz geeignet. Erhältlich ist das Gel in den Größen 150 ml (für die Kitteltasche), 500 und $1000 \mathrm{ml}$. Es kann auch einzeln als Praxisbedarf bestellt werden: Tel. 0800/86 87 87-0 oder www.roggverbandstoffe.de. ROGG 